Retos, I0(19), 2020

\title{
La cooperación entre firmas en el desarrollo regional: una revisión
}

\section{Cooperation between firms and regional development: a review}

Sofía Carpio es investigadora de la Facultad Latinoamericana de Ciencias Sociales, FLACSO (Ecuador) (stcarpio8@gmail.com) (https://orcid.org/0000-0003-2350-3041)

\section{Resumen}

El estudio de la cooperación entre empresas dentro del campo del desarrollo económico tiene una amplia trayectoria en el área del desarrollo regional. El presente trabajo realiza una revisión de literatura sobre el papel de la cooperación entre firmas dentro de las experiencias de desarrollo regional. Se incluyeron trabajos que, mediante la revisión de casos empíricos, identificaban los factores que explican por qué en algunos casos emergen relaciones de cooperación entre firmas mientras que, en otros, no. Se encontraron varias similitudes en las dimensiones estudiadas: las condiciones que ofrecen las aglomeraciones para la interacción repetida entre las firmas, la necesidad de mecanismos de gobernanza para evitar comportamientos oportunistas, la institucionalización de la cooperación, el efecto de factores externos a la región sobre la continuidad de los procesos de cooperación. También se identificó un énfasis en los procesos de aprendizaje, diseminación de conocimiento e innovación que ocurren en las configuraciones industriales observadas usualmente en los clústeres, distritos industriales o región. Se resume el papel de la cooperación en el desarrollo regional en cuatro dimensiones principales: 1) la organización industrial de los distritos industriales, clústeres y regiones; 2) el equilibrio entre procesos de cooperación y competencia; 3 ) la gobernanza y la construcción de relaciones de confianza y reciprocidad; y 4) el efecto de factores externos sobre la cooperación entre firmas, otorgando a estas relaciones un carácter dinámico.

\begin{abstract}
The study of cooperation between firms within the field of economic development has a longstanding tradition, particularly in regional development. The present work seeks to carry out a review of the literature concerned with the role firm cooperation plays in regional development. The review includes works that analyze empirical cases and aim to identify the elements that explain why in some cases inter-firm cooperation is successful while in others, it is not the observed outcome. The dimensions of cooperation emphasized in the surveyed works share various similarities: the conditions in regional agglomerations that enable repeated interactions between firms to take place, the need for governance mechanisms to enforce cooperation between firms and to prevent opportunistic behavior from arising, the institutionalization of cooperation, the impact external factors have on the continuation of cooperation between firms. An emphasis in the literature on learning, knowledge-diffusion and innovation processes was identified. In the concluding remarks, the role of cooperation between firms in the regional development literature was summarized in four main dimensions: 1) the industrial organization of industrial districts, clusters and regions; 2) the balance between cooperation and competition; 3) governance mechanisms and the building of trust and reciprocity in firm relations; and 4) the impact of external factors on inter-firm cooperation, rendering these relations dynamic.
\end{abstract}

\section{Palabras clave I keywords}

Cooperación entre firmas, desarrollo regional, distritos industriales, clústeres, organización industrial Interfirm cooperation, regional development, industrial districts, clusters, industrial organization

Cómo citar: Carpio, S. (2020). La cooperación entre firmas en el desarrollo regional: una revisión. Retos Revista de Ciencias de la Administración y Economía, 10(19), 117-133. https://doi. org/10.17163/ret.n19.2020.07 


\section{Introducción}

La cooperación en el campo de la economía del desarrollo ha sido frecuentemente estudiada en el desarrollo regional y la economía geográfica. Este es el caso, en particular, a partir del éxito de algunos casos de desarrollo regional específicos, en los que se observaron procesos de producción en redes de PYMES que interactuaban por medio de arreglos de cooperación y competencia, alcanzando una alta competitividad global. Entre los casos que más atención recibieron a nivel internacional se encuentran la Tercera Italia, Alemania y otros distritos industriales europeos, y también el caso de Japón cuya organización industrial, a diferencia de otros países industrializados, se caracteriza por un gran porcentaje de pequeñas y medianas firmas (Sayer, 1989; Carlsson, 1989). Surgieron varios trabajos dedicados al estudio de los distritos industriales, el "efecto distrito" y los clústeres como una forma de promover el desarrollo económico regional y también, nacional. Dentro de estos trabajos se pueden identificar varias líneas de investigación (las economías externas y la eficiencia colectiva en los distritos industriales, la industrialización a escala pequeña, la innovación en redes o clústeres productivos, las relaciones industriales, solo por mencionar algunas). A juzgar por la cantidad de perspectivas desde las cuáles se puede abordar el tema de la cooperación, las relaciones entre firmas, los distritos industriales y el desarrollo económico en sí, investigar el vínculo entre la cooperación dentro del campo de la economía del desarrollo requiere delimitar el abordaje, aceptando que habrá aristas que quedarán por fuera, mientras que a otras se les otorgará un mayor énfasis.

Tanto las líneas de investigación que se muestran arriba, como el contexto en el cual surge esta relación entre la cooperación y el desarrollo regional, indican que existe un vínculo importante entre la cooperación entre firmas y su escala. Es decir, en la presente revisión se privilegia el estudio de la cooperación entre firmas de escala reducida o, la cooperación entre firmas grandes y firmas pequeñas, estas últimas siempre presentes en la ecuación. De esta observación se puede esbozar otro vínculo entre el estudio de la cooperación entre firmas y el desarrollo, específicamente, un tipo de desarrollo cuyos beneficios están distribuidos de forma más equitativa. El estudio de los procesos en los que grupos o redes productivas de PYMES logran cooperar para alcanzar competitividad internacional y promulgar procesos de desarrollo regional es importante para la formulación de estrategias y políticas públicas que promuevan un desarrollo más equitativo, a nivel regional y nacional.

Con este objetivo en mente, el presente trabajo busca realizar una revisión de literatura del papel de la cooperación entre firmas dentro de experiencias de desarrollo regional. Por un lado, se busca abordar la revisión desde un punto de vista teórico, seleccionando trabajos que abordan la conceptualización de la cooperación entre firmas y tratan de explicar las condiciones bajo los cuales esta es exitosa. Por otro lado, se busca revisar trabajos que revisen casos específicos. A continuación, se resumen los hallazgos en la discusión académica en torno a este tema, los debates y consensos encontrados.

\section{Metodología: criterios de selección}

Para la selección de los trabajos, parte de esta revisión de literatura, se utilizaron las herramientas Scopus y Google Scholar, empleando los siguientes términos en la búsque- 
da: "cooperation in economic development", "firm cooperation in economic development", "Cooperation in development economics", "interfirm cooperation development". En la selección de trabajos se priorizaron aquellos que abordaban conceptualmente el estudio de la cooperación entre firmas, así como experiencias específicas de desarrollo regional. Algunos trabajos se seleccionaron de entre aquellos referenciados por la selección inicial cuando éstos parecían ser bastante relevantes al enfoque delineado en el presente trabajo para abordar el vínculo entre cooperación y desarrollo. Este fue el caso, en particular, en la búsqueda de abordajes teóricos de la cooperación entre firmas.

\section{Resultados y discusión}

La cooperación entre firmas ha sido abordada en varios trabajos en la literatura de distritos industriales, clústeres y desarrollo regional. En esta revisión, se introducen los tipos de cooperación identificados en las relaciones entre firmas, al tiempo que se resumen las conceptualizaciones teóricas de cada autor, identificando similitudes y diferencias.

\subsection{La cooperación entre firmas en la literatura de desarrollo económico}

La cooperación entre firmas en el campo del desarrollo económico cobra especial relevancia a partir de la experiencia de los distritos industriales en la Tercera Italia y posteriormente, la identificación de distritos industriales en otras partes de Europa y del mundo (Rabelloti, 1997; Schmitz, 1995; Amin, 1994). Al mismo tiempo, desde los estudios posfordistas surgen varias corrientes que identifican un cambio en la forma de organización de la producción industrial en las principales economías industrializadas (Piore \& Sabel, 1984; Acs \& Audretsch, 1990). En un contexto de cambio tecnológico, se percibía un distanciamiento de una organización industrial jerárquica, de integración vertical, producción estandarizada y masificada (denominada producción Fordista) hacia una organización industrial más descentralizada, en escalas más pequeñas, flexible y heterárquica (Piore \& Sabel, 1984; Cooke, 1998). Los trabajos que abordan esta transformación desde distintas corrientes teóricas abundan y persisten algunos debates al respecto (ver Amin, 1994).

En general, esta forma emergente de organización industrial se caracteriza por la conformación de redes productivas de firmas pequeñas y medianas, o alternativamente, de firmas grandes que empiezan a subcontratar varias de sus actividades a PYMES para concentrarse en sus competencias nucleares (Loveman \& Sengenberger, 1991) con las cuales establecen relaciones más cooperativas con el fin de responder a una demanda fluctuante de productos diferenciados y mercados más segmentados (Sabel, 1994). Sin embargo, relevante para la presente revisión es la conclusión de que, bajo esta nueva forma de organizar la producción industrial en un contexto de globalización que se profundiza, las relaciones de cooperación y competencia entre firmas para construir y mantener una ventaja competitiva cobran fundamental importancia (Raco, 1999; Cooke, 1998). Ya sea en el contexto de redes productivas de empresas que operan en aglomeraciones geográficas o en un contexto de firmas grandes que incrementan la subcontratación de firmas pequeñas y medianas para la realización de algunas de sus actividades (Sabel, 1994), en ambos casos, se vuelve 
imperativa la necesidad de cooperar para lograr una coordinación de toda la cadena de valor de manera que sea posible mantener (o construir) competitividad.

Dentro de la literatura de desarrollo regional, surgen varios trabajos que buscan construir una formulación teórica que explique por qué en algunos casos (y bajo qué circunstancias) estos procesos de cooperación son exitosos, y por qué en otros, no. En la siguiente sección se introducirán los conceptos derivados de estas formulaciones teóricas. Después de introducir el concepto de economías externas, los procesos de cooperación deliberada entre firmas se pueden dividir en: la provisión de bienes públicos, la coordinación de actividades productivas y otras formas de cooperación.

\subsection{Las economías externas de Marshall}

Las economías externas son quizás la primera formulación teórica mediante la cual se buscó explicar la competitividad que adquirían aquellas firmas aglomeradas geográficamente y que se agrupaban entorno a una misma actividad económica (Rabellotti, 1997; Schmitz, 1995). Las economías externas de los distritos industriales contribuyen a disminuir los costos de transacción entre las firmas. Constituyen efectos secundarios de las transacciones de mercado entre las firmas y tienen un carácter de bien público que hace que su provisión no se dé al nivel óptimo. En otras palabras, al ser externalidades positivas, las firmas que las producen no lo hacen al nivel en el que el costo social marginal equivale a la ganancia social marginal de producción. Es en este punto en donde la cooperación entre firmas es el elemento que explica cómo se pueden aprovechar de mejor manera las economías externas mediante la provisión de bienes públicos a niveles óptimos y evitando el problema del polizón (Rabelloti, 1997, pp. 33-36).

\subsection{Los bienes públicos y la eficiencia colectiva}

Otra ventaja que se puede encontrar en algunos casos de aglomeraciones productivas y que está presente en la mayoría de los trabajos revisados es la posibilidad de cooperar con el fin de financiar ciertos bienes y servicios que beneficien a todas las firmas, es decir, la posibilidad de ponerse de acuerdo para producir bienes públicos. Estos pueden incluir los siguientes: facilidad de acceso a financiamiento, la posibilidad de comprar materia prima conjuntamente, I + D, marketing, servicios de inteligencia de mercado, seguro de desempleo, institutos técnicos de entrenamiento y educación para trabajadores, entre otros (Lorenz, 1992, p. 175) (Enright, 1995 en Raco, 1999, p. 953; Rabelloti, 1997; Schmitz, 1995; Porter, 1990, pp. 80-81). Lograr cooperar para producir este tipo de bienes facilita a las PYMES acceder a ciertos servicios que usualmente están reservados para empresas grandes, debido a su costo. Generalmente, la provisión de estos servicios está mediada por alguna institución creada para ese fin. Esta puede ser privada (una organización gremial, un instituto técnico cofinanciado de forma pública y privada, un banco o cooperativa) o pública (Rabelloti, 1997, p. 4; Arku, 2014; Goetz, 1993; Gordon, 2007). Existe toda una literatura que estudia a estas instituciones y mecanismos, públicos o privados, locales o nacionales. Estos trabajos no forman parte de esta revisión de literatura, que se enfoca más bien en los mecanismos de cooperación entre firmas y no en el papel que puedan jugar otras instituciones públicas o privadas en el proceso; sin embargo, es importante recalcar 
que un aspecto de la cooperación en las relaciones entre firmas es, justamente, como se verá más adelante, la dimensión de gobernanza de estos procesos.

Schmitz (1995) agrupa el concepto de economías externas, la provisión de bienes públicos y otras formas de cooperación entre firmas dentro de un solo concepto, la eficiencia colectiva, definida como "la ventaja competitiva que se deriva de las economías externas locales y la acción colectiva” de firmas concentradas geográfica y sectorialmente (Schmitz, 1995, p. 530). La acción colectiva involucra las acciones de cooperación expresas (y no secundarias) que llevan a cabo las empresas. Esta cooperación puede tomar varias formas, ya sea como colaboración entre firmas específicas (por ejemplo, en proyectos de innovación o adopción de tecnología, coordinación entre firmas y sus subcontratistas), o a través de instituciones públicas y privadas que proveen servicios a todas las firmas del distrito (como la provisión de entrenamiento técnico, servicios de inteligencia de mercado, acceso a financiamiento, entre otros). Dentro de este último se encuentra la cooperación para la provisión de bienes públicos. Mientras las economías externas son un efecto no planificado, un producto secundario de las interacciones entre las firmas del distrito, la acción colectiva consiste en un esfuerzo colectivo consciente (Schmitz, 1995, p. 536). El autor recalca la distinción entre aquellos procesos de cooperación pasivos (las economías externas) y aquellos que requieren de la proactividad de las firmas (bienes públicos y los otros procesos de cooperación) (Schmitz, 1995, p. 536).

\subsection{Otras formas de cooperación}

Existen otros procesos de cooperación que son indispensables para incrementar la competitividad del distrito, clúster o región. Estos procesos incluyen, por un lado, acciones encaminadas a coordinar la producción en toda la cadena de valor, y por otro, la adhesión a ciertas normas acordadas de competencia (Lorenz, 1992; Cooke, 1998, p. 22). En esta última intercede un balance entre cooperación y competencia, que deviene en un proceso necesario para construir e incrementar la ventaja competitiva en toda la aglomeración; sobre este punto se profundizará en la siguiente sección. A continuación, se ahonda sobre la necesidad de coordinar las actividades en toda la cadena o complejo de valor.

Debido a que la producción se organiza en una cadena de valor en la que participan varias firmas, se vuelve imperativo que en cada eslabón se den procesos de coordinación para asegurar que las especificaciones de los bienes que produce cada firma sean las adecuadas, con el fin de garantizar la calidad en toda la cadena, posibilitar una respuesta rápida (y flexible) de la producción ante fluctuaciones en la demanda final, cambios en gustos diferenciados y por último, para impulsar procesos de innovación en toda la cadena que le permitan mantener su competitividad. A esta configuración de la producción industrial localizada de carácter más complejo Patchell (1996) denomina el "complejo de valor regional”. Este se distingue de la "cadena de valor regional" en que, en lugar de existir una sola firma nuclear que subcontrata varios de sus procesos y vende sus productos a través de varios distribuidores (una cadena de valor), existen varias firmas nucleares que a su vez subcontratan varios de sus procesos a otras firmas, y distribuyen por medio de varios distribuidores 
(Patchell, 1996, p. 490). En general, este es el caso de varios de los distritos industriales identificados.

Figura 1. Complejo regional de valor con rivalidad entre subcontratistas, firmas nucleares, distribuidores y compradores

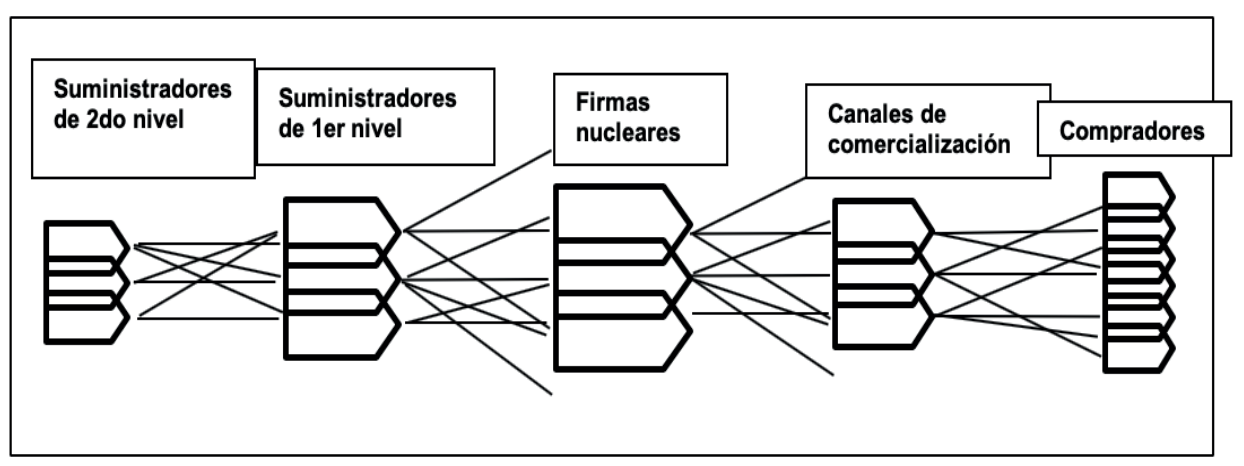

Fuente: Patchell 1996, p. 490.

Una forma de organización industrial similarmente compleja se puede encontrar en la experiencia de Japón, en Sayer (1989). Como se puede observar en la Figura 2, las actividades económicas que componen la cadena de valor están repartidas entre varias firmas, en un arreglo bastante desintegrado verticalmente. El caso de Japón se diferencia de las otras experiencias mencionadas en los trabajos revisados en que, existen firmas grandes que se encuentran en la cima de la pirámide organizacional. Sin embargo, estas subcontratan gran parte de sus actividades a otras firmas con las que establecen relaciones en las que los procesos de cooperación y coordinación son tan esenciales como en el caso de los distritos industriales y clústeres compuestos por redes de PYMES. Existe todavía cierta jerarquía; sin embargo, la necesidad de que existan relaciones de confianza que posibiliten la cooperación y coordinación entre las firmas es un requisito para una producción más flexible capaz de responder a fluctuaciones en la demanda internacional y la constante innovación y desarrollo de productos (Sayer, 1989). Otros recuentos del modelo japonés, por ejemplo, en la industria de semiconductores, recalcan la naturaleza jerárquica del modelo y matizan el nivel de cooperación existente entre firmas que compiten horizontalmente; sin embargo, el papel de la cooperación entre firmas en el surgimiento de la competitividad japonesa en esta industria específica fue reconocido a nivel mundial al punto de propiciar iniciativas de cooperación en investigación en otros países productores de semiconductores (Estados Unidos y Gran Bretaña) que vieron su participación en el mercado internacional mermado por la intromisión japonesa (Thornberry, 2002, p. 665). 
Figura 2. Tipos de grupos industriales en Japón

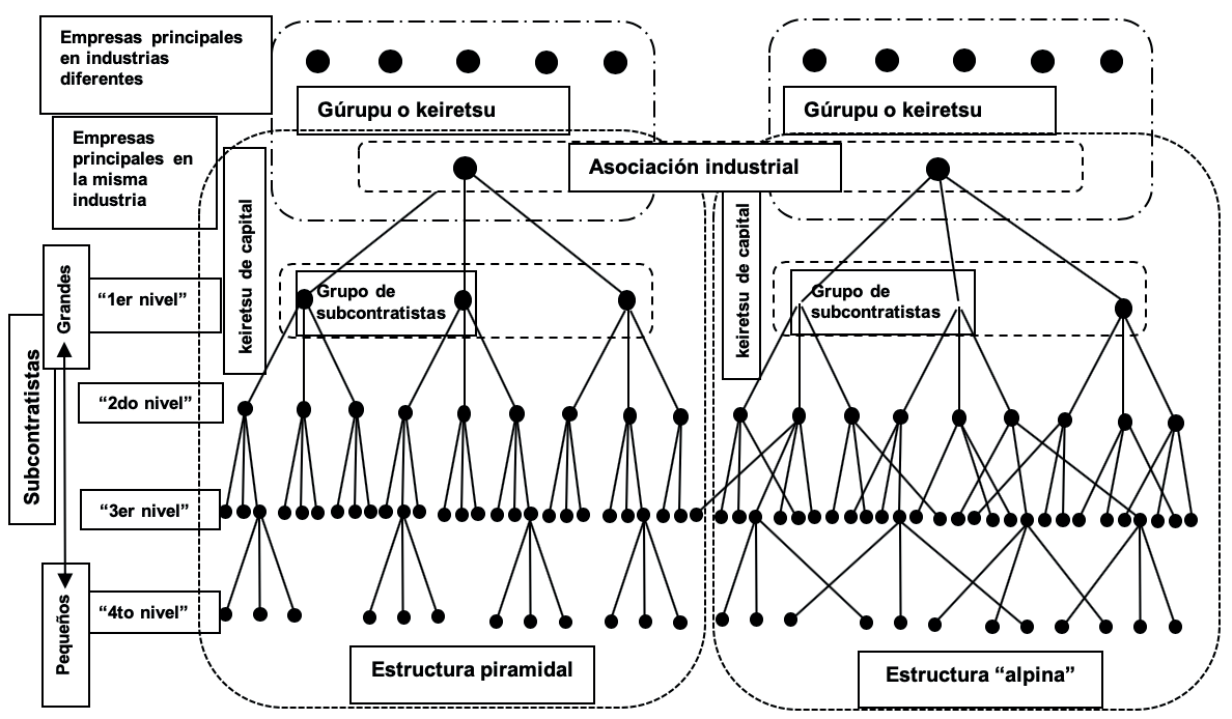

Fuente: Sayer, 1989, p. 684.

En este contexto de un movimiento de la organización industrial mundial hacia la desintegración vertical, una producción más flexible y la complejización de las cadenas de valor, así como el surgimiento de un mayor número de relaciones de interdependencia entre las firmas, la necesidad de construir y mantener relaciones de cooperación entre las firmas que componen la cadena es evidente.

Hasta el momento se han mencionado las economías externas, la provisión de bienes públicos y la coordinación de actividades en toda la cadena (o complejo) de valor. Sin embargo, existen otras formas de cooperación que involucran el respeto de ciertas "normas de competencia" ante situaciones cambiantes (Lorenz, 1992, p. 179; Cooke, 1998, p. 22). A continuación, se describen algunas de las conceptualizaciones teóricas de cada autor para explicar las relaciones cooperativas entre firmas, después de lo cual se resumen las similitudes y diferencias entre ellas.

\subsection{Conceptualizaciones teóricas de las relaciones de cooperación entre firmas}

En los trabajos revisados que abordan la conceptualización teórica de la cooperación se encontraron términos distintos, dependiendo desde qué literatura se aborda el tema; sin embargo, en todos los casos se hace referencia a un balance entre competencia y cooperación y se recalca que estos comportamientos no son opuestos, sino que conforman una dinámica en las relaciones entre firmas.

Patchell (1996) se basa en la definición de Axelrod (1984) de cooperación, quien utiliza teoría de juegos para explicar por qué en las interacciones entre firmas pueden triunfar las estrategias cooperadoras sobre las estrategias no cooperadoras. 
Según Axelrod "la cooperación es definida como una relación voluntaria en la que entran las partes en busca de beneficios egoístas que son mutuos y cuya evolución es promovida por la localización de una población" (Axelrod, 1984 en Patchell, 1996, p. 481). Basándose en teoría de juegos, específicamente el Dilema del Prisionero con varias iteraciones, Axelrod (1984) investiga las relaciones de cooperación (o deserción) entre firmas. El autor encuentra que las estrategias cooperadoras pueden invadir exitosamente a una población de estrategias no cooperadoras. Estos resultados han sido replicados por otros autores (Nowak, 2006, pp. 71-91).

Según Patchell (1996), la cooperación entre firmas sirve para mediar entre la competencia y el control. Por control, el autor se refiere al control sobre la proporción de beneficios que le corresponde a cada firma que participa de una transacción económica. En este contexto, cooperar implica la capacidad de cada firma de ceder a la otra firma parte del control sobre qué proporción de los beneficios totales le corresponde (Patchell, 1996, p. 487). Para ello, debe existir un nivel de confianza que permita a cada firma exponerse a que la(s) otra(s) firma(s) adopte(n) un comportamiento oportunista y que aun así el desenlace sea uno en el que las relaciones mantengan su carácter cooperativo. Para el autor, para cada uno de estos elementos (cooperación, control, competencia) existe una situación en la que, de no existir un balance e incurrir en un exceso, se puede caer en un escenario indeseado. Un exceso de cooperación entre las firmas puede llevar a la colusión entre ellas de manera que se asfixie la competencia horizontal o vertical, se promuevan asignaciones no basadas en criterios de eficiencia y se pierda competitividad. Una competencia exacerbada puede llevar al tipo de rivalidad exacerbada que, al transformarse en una carrera por recortar costos, pueda resquebrajar la confianza establecida y afectar la coordinación en toda la cadena. Por último, un nivel de control desbalanceado puede convertirse en una represión en la que algunas firmas abusen de su poder en detrimento de las demás, afectando las redes de confianza y cooperación construidas (Patchell, 1996, pp. 484-485). Es así como la cooperación es entendida como un mecanismo mediador entre un nivel (y tipo) de competencia deseados y el control sobre los beneficios generados.

Desde la sociología y sociología económica, Trapido (2007) define a la cooperación empleando el concepto de interdependencia (Emerson, 1962 en Trapido, 2007). En su definición, la competencia consiste en una lucha entre dos firmas por los recursos de un tercero y la medida en que esta lucha la llevan a cabo de manera independiente (pp. 166-167). La cooperación consiste entonces en el manejo de la interdependencia que se da sobre un espectro continuo donde, en un extremo se encuentra la competencia puramente independiente entre firmas y en el otro, la fusión de estas; en el medio se encuentran las acciones en las que las firmas cooperan para obtener sus objetivos (p. 167).

A pesar emplear términos distintos, ambas conceptualizaciones reconocen un grado de interdependencia entre las firmas como agentes económicos y perciben el proceso de cooperación como un balance entre una competencia pura y una colaboración entre ambos agentes. Esta noción se encuentra también en la revisión de literatura realizada por Newlands (2002), en donde es una de las tres dimensiones empleadas para comparar las distintas corrientes teóricas sobre clústeres industriales. 
En la misma línea, Lorenz (1992, p. 179) alude a la cooperación como la adhesión a ciertas "normas de competencia". Esta constituiría una tercera forma de cooperación identificada en la literatura de desarrollo regional, junto con la provisión de bienes públicos y las actividades de coordinación de la producción en toda la cadena, mencionadas anteriormente. La adhesión a ciertas normas de competencia busca evitar que surja un tipo de competencia cuello-a-cuello entre firmas, un juego de suma-cero, o lo que Trapido (2007) denomina una "interdependencia negativa"; es decir, una situación en la que ambas firmas perciben que sus beneficios son directamente proporcionales a las pérdidas la otra (Trapido, 2007, p. 166). Las "normas de competencia" toman otros nombres al tomar conceptos de la literatura de acción colectiva, como se ve en Cooke (1998).

Otro concepto utilizado para explicar los distintos tipos de competencia es la distinción entre lo que se ha denominado un camino "alto" (o "noble") y un "camino bajo" (o "menos noble") de competencia entre empresas (Schmitz, 1995, p. 541; Loveman \& Sengenberger, 1991, p. 27; Ornston, 2014). El "camino noble" se refiere a procesos de competencia que se dan a través de mejoras en la calidad, innovación de productos, disminución en tiempo de respuesta y demás dimensiones que no involucran lo que sería la competencia "menos noble" que, en cambio, implica la disminución de precios, flexibilización laboral, empleo barato y la utilización de materiales de menor calidad (Schmitz, 1995, p. 541). En la revisión de literatura llevada a cabo por Schmitz de casos de distritos industriales en países en vías de desarrollo, es frecuente encontrar la aplicación de distintos grados de ambos tipos de competencia (Schmitz, 1995, pp. 541-542).

En las aglomeraciones industriales, este tipo de competencia cuello-a-cuello basada primordialmente en recortar costos como sea para ganar una mayor proporción del mercado conlleva a una "disminución de precios, robo de personal capacitado de las otras firmas, un círculo vicioso de falta de inversión y una depreciación y devaluación del trabajo" (Florida \& Kenney, 1990 en Patchell, 1996). El tipo de competencia deseado en las relaciones entre firmas es una "competencia interdependiente" o "competencia horizontal" donde cada firma emplea distintas estrategias genéricas (Porter, 1985) y se beneficia de las ventajas derivadas de que toda la industria exhiba competitividad: atraer inversiones, desalentar la entrada de nuevos competidores al mercado, contribuir al desarrollo de mercado y mejorar la estructura de la industria (Porter, 1980 en Patchell, 1996, p. 489). Se busca propiciar una competencia mediante procesos de innovación, mejora de la calidad, segmentación y especialización de la oferta, de manera que se impulse mejoras en la calidad, desarrollo de nuevos productos y la complejización de la producción, en beneficio de la competitividad colectiva de toda la aglomeración. Mantener este tipo de competencia requiere que las firmas adopten ciertas normas de competencia e incluso, de enfrentarse a circunstancias internacionales desfavorables, se apoyen en épocas de disminución de la demanda efectiva externa. Manteniendo el balance cooperación-competencia, en esta afirmación no se desestima la necesidad de que exista "rivalidad local" que incentive a invertir en procesos de innovación y mejoras constantes (Porter, 1990, p. 80).

La adhesión a ciertas normas de competencia hace posible evitar que se exploten vulnerabilidades de la cadena que favorezcan el oportunismo individual de las firmas. Ante las circunstancias cambiantes de la demanda internacional, la falta de 
confianza y cooperación entre las firmas podría traducirse en oportunidades para que las firmas prioricen su beneficio individual de corto plazo y perjudiquen al resto de firmas de la red. Si una o más firmas adopta un comportamiento oportunista, se rompen las redes de confianza, en detrimento de toda la cadena o complejo de valor, la eficiencia y competitividad colectivas. Similarmente, la necesidad de cooperar es evidente también en cadenas de valor en las que participan firmas grandes y sus subcontratistas, de manera que se garantice la supervivencia de todos en el mercado. En este caso, la cooperación hace posible que, en lugar de escudarse en sus subcontratistas ante demandas fluctuantes, las firmas grandes los apoyen. La experiencia de Japón es un ejemplo de relaciones entre firmas de colaboración estratégica en lugar de explotación (Loveman \& Sengenberger, 1991; Sayer, 1989).

Por último, Rabelloti (1997) identifica otra dimensión de la eficiencia colectiva que explica la heterogeneidad observada en el desempeño de las firmas y su utilización de los mecanismos de cooperación disponibles en el distrito. Dentro de las acciones colectivas de cooperación, existen ciertas acciones que benefician a una gran proporción de las firmas del distrito (sino a todo el distrito) y cuyos beneficios no son excluibles. Pero, también existen otras acciones cuyos beneficios son excluibles y que se podrían considerar "bienes club" en el sentido de que solo las firmas que cooperan reciben sus beneficios. De esta manera, funcionan como una recompensa por haber cooperado, e incrementan los incentivos para cooperar. Ejemplos incluyen: intercambio de información, tecnología, know-how o capital humano, intercambio monetario, reputación que sirva en futuras interacciones con las firmas del distrito (Rabellotti, 1997, p. 39). Por último, la capacidad de cada firma de aprovechar el acceso a los beneficios derivados de las acciones de cooperación explicaría también esta heterogeneidad (pp. 39-40).

\subsection{Factores que posibilitan el surgimiento de relaciones de cooperación entre firmas}

¿Qué factores explican el surgimiento y sostenimiento de estos tipos de cooperación entre firmas (la provisión de bienes colectivos, coordinación de actividades en la cadena, adherencia a ciertas normas de competencia) en las experiencias de desarrollo regional revisadas? En la siguiente sección se identifican los factores que se mencionan en la literatura, mismos que se repiten en la gran mayoría de trabajos revisados.

En concordancia con los hallazgos de Axelrod (1984), para que las firmas opten por mantener un comportamiento cooperador, estas deben anticipar que la interacción con la(s) otra(s) firma(s) sea repetida. Este es el caso de las firmas en una aglomeración ya que, al encontrarse localizadas geográfica y sectorialmente, se prevé que su interacción con las demás firmas se repita a futuro. Por tanto, para cada firma, los beneficios esperados de adoptar un comportamiento cooperador son mayores en el largo plazo que la alternativa de adoptar un comportamiento oportunista que traería beneficios de corto plazo (Lorenz, 1992; Rabellotti, 1997, pp. 37-38). A esta situación, Patchell denomina la restricción espacio-temporal (Patchell, 1996, p. 492). El aspecto temporal de la restricción está dado por el carácter de relaciones comerciales de larga trayectoria entre los actores económicos, que es el caso en varias 
de las experiencias revisadas (Rabelloti, 1997, p. 38; Sayer, 1989; Piore \& Sabel, 1984; Kamnungwut \& Guy, 2012).

El segundo factor para alcanzar un resultado cooperador es la existencia de reciprocidad (Patchell, 1993, p. 493; Schmitz, 1995, p. 541). Aquellas firmas que eligen no cooperar deben esperar una retaliación por parte del resto del grupo; esta pérdida de beneficios actúa como un desincentivo para adoptar un comportamiento oportunista. Esta observación está presente en la aplicación del Dilema del Prisionero a este contexto (Axelrod, 1984). Las estrategias más exitosas fueron aquellas que replicaban el comportamiento del otro jugador; es decir, una vez que el otro jugador (o alguno de los otros jugadores) elije no cooperar, la firma deja de cooperar también (Axelrod, 1984 en Patchell, 1996, pp. 485-486). Algo similar se encuentra en los trabajos seleccionados que revisan casos empíricos, en los que se menciona la necesidad de sancionar a las firmas que no se adhieren a las "normas de competencia" establecidas, en la mayoría de los casos, informalmente. Lorenz (1993) menciona a la sanción social como un mecanismo de control social que asegura el mantenimiento de la cooperación en una comunidad. En su investigación sobre la cooperación de distintos actores locales para atraer inversiones privadas a localidades en Ohio (Estados Unidos), Cox y Wood (1997) mencionan la importancia de la confianza en la red de relaciones entre los actores vinculados al desarrollo económico local y el conocimiento generalizado de que una traición a esta confianza ("código de honor") incurriría en la exclusión de procesos futuros de atracción de inversiones (Cox \& Wood, 1997, p. 83).

Lorenz (1992) menciona otra observación ausente en el resto de los trabajos revisados: la necesidad de que existan mecanismos que puedan redistribuir de forma más equitativa los beneficios obtenidos por todo el complejo productivo (leveling mechanisms), que pueden incluir seguro de desempleo, redistribución de la demanda externa hacia firmas menos exitosas y acceso a crédito (Lorenz, 1992, p. 179). Según el autor, el incremento de la desigualdad económica entre las firmas de una aglomeración puede provocar desconfianza y el cuestionamiento de la interdependencia de toda la red. Al mismo tiempo, si estos mecanismos resultan en una redistribución excesiva, pueden resultar en desincentivar la inversión en innovaciones y mejoras. Por tanto, se debe encontrar un balance entre recompensar las iniciativas de emprendimiento sin que la desigualdad en toda la aglomeración crezca desmesuradamente. En este sentido, la necesidad de un sistema de gobernanza adecuada del que participen todas las firmas se vuelve más evidente (Lorenz, 1992). Ornston (2014) reporta un crecimiento en la desigualdad de la fuerza laboral (entre trabajadores de cuello blanco y azul) en el proceso de resquebrajamiento de las relaciones de cooperación en la industria finlandesa (Ornston, 2014, p. 460).

La predisposición a mantener relaciones de cooperación con otras firmas depende la información que tenga cada firma y sus creencias acerca del comportamiento del resto de integrantes del clúster, distrito o red. Si las firmas creen que el resto de las empresas van a adoptar comportamientos oportunistas, entonces optarán por hacer lo mismo, imposibilitando el surgimiento de procesos de cooperación. Por tanto, para suscitar relaciones cooperativas, se requiere de la existencia de cierto nivel de confianza entre las firmas. En todos los trabajos revisados se identifica la confianza como un tercer factor determinante en la cooperación exitosa entre firmas. 
La presencia de lazos comunitarios fuertes es uno de los factores frecuentemente citados en la literatura para explicar la existencia de redes de confianza y surgimiento de procesos de cooperación sostenidos. En comunidades donde los miembros comparten valores, creencias, ciertos rasgos culturales que los vuelven una unidad territorial con un tejido social sólido, es más fácil que predomine un alto nivel de confianza. En Rabellotti, este aspecto es una de las características que separa a un distrito industrial del resto de tipo de aglomeraciones geográficas: la existencia de "un trasfondo social y cultural fuerte y relativamente homogéneo que vincula los agentes económicos y cree un código de comportamiento común, a veces explícito, pero frecuentemente implícito, y ampliamente aceptado" (Rabellotti, 1997, p. 23).

La existencia de:

Un capital social de tipo cultural, comunal, étnico o de índole religiosa que produce una sanción lo suficientemente fuerte como para prevenir un rompimiento de las normas en primer lugar, o para proveer una base lo suficientemente fuerte como para reparar ese resquebrajamiento. (Cooke, 1998, p. 18)

Al comparar las experiencias de Silicon Valley con la Ruta 128, ambos, clústeres tecnológicos en Estados Unidos, Trapido (2007) destaca en la primera la existencia de una cultura de negocios abierta a la cooperación como un factor que posibilita el intercambio de conocimiento entre firmas; mientras, la segunda experiencia es reconocida por su reticencia a este tipo de cooperación (Saxenian, 1994 en Trapido, 2007, p. 170). Una de las hipótesis que examina el autor en su estudio de la cooperación en el mercado de capitales de riesgo es justamente la hipótesis cultural de surgimiento de la cooperación (Trapido, 2007, p. 170). Así mismo, Felzensztein (2008) identifica una mayor cohesión social y "colectivismo" como un factor que facilita la cooperación entre firmas en actividades de marketing (p. 240). Neto (2008) menciona entre las barreras para cooperación en América Latina la falta de confianza y una tradición de cooperación.

Sin embargo, existe también evidencia de contextos de cohesión cultural que dificultan el surgimiento o profundización de la cooperación entre firmas. Patchell (1996) menciona un caso así en Guangdong (China) donde las relaciones entre firmas están fuertemente atravesadas por lazos de parentesco al punto que se limita la entrada de otras firmas y, por tanto, la competencia horizontal que equilibre la colaboración entre firmas. Así mismo, Schmitz (1995) reporta un caso similar en un distrito de producción de calzado en Agra (India) (Knorringa, 1994 en Schmitz, 1995, p. 541). El papel que juegan las redes de confianza en el balance entre competencia y cooperación no está del todo resuelto; sin embargo, la evidencia es contundente con respecto a su importancia en el surgimiento de la cooperación entre firmas.

Otros trabajos revisados hacen alusión a la existencia de redes de confianza construidas no a partir de un bagaje cultural compartido, sino de interacciones pasadas repetidas entre las firmas. Utilizando data del mercado de capitales de riesgo en Estados Unidos, Trapido (2007) confirma la hipótesis de que empresas que en el pasado fueron competidores son más propensas a cooperar en el futuro. Su explicación yace en que, al haber competido en el pasado, las firmas se percatan de la existencia de la otra, interactúan de manera repetida, lo que conlleva a que se conozcan 
personalmente y puedan construir un nivel de confianza que posibilite acciones de cooperación a futuro (Trapido, 2007, p. 169). Otro caso similar en la industria de semiconductores estadounidense se describe en Thornberry (2002), en la cooperación en actividades de investigación; en este último ejemplo, existen algunas variables específicas a este caso que contribuyeron a su éxito.

Cierto grado de confianza permite que parte de la coordinación pueda ser llevada a cabo mediante acuerdos informales, lo cual le adjudica a todo el complejo productivo la flexibilidad para cambiar aspectos de la producción de surgir la necesidad, sin tener que preocuparse por las rigideces de contratos formales (Patchell, 1996, p. 494; Acs \& Fitzroy, 1989, p. 313). A medida que estos arreglos se mantienen y respetan, la cadena (o complejo) de valor va construyendo una ventaja competitiva ya que posee un nivel de coordinación que le otorga flexibilidad, y se pueden tomar los riesgos necesarios para realizar inversiones e involucrarse en procesos de innovación (Lorenz, 1992, p. 180).

Otro aspecto recurrente en la literatura de cooperación entre firmas en experiencias de desarrollo regional es la institucionalización de la cooperación y mecanismos de gobernanza para su mantenimiento en el tiempo. Al respecto, Patchell (1996) enfatiza sobre la necesidad de un mecanismo de control externo a la firma, en el que las decisiones sobre aspectos de la producción y el control de la proporción de beneficios correspondientes a cada firma no sea interno a una firma nuclear, sino que sea compartido entre las firmas involucradas en las transacciones: "A medida que la producción se vuelve más sofisticada, la necesidad de garantizar una gobernanza externa del desarrollo, calidad, costo, distribución e incremento de las ganancias se vuelve más importante" (Patchell, 1996, p. 491). Raco en cambio, menciona las ventajas de institucionalizar los mecanismos de cooperación a través de la asociatividad como un medio para profundizar los mecanismos de cooperación; su trabajo se enfoca en el aspecto de aprendizaje constante y diseminación de información como fuente de competitividad del clúster, por lo que este tipo de institucionalización generaría canales adicionales para el aprendizaje colectivo (Raco, 1999, pp. 954-955). La literatura enfocada en este aspecto y también en las acciones que pueden emprender estas instituciones para fomentar la cooperación entre firmas, así como políticas públicas dirigidas a promover el desarrollo regional a partir de la cooperación entre actores económicos es bastante amplia.

\subsection{La región innovadora y las economías aprendientes}

En este punto, vale mencionar otro enfoque identificado en la literatura que se centra sobre los procesos de innovación y aprendizaje regionales, que le otorgan un dinamismo tecnológico a la región y que son resultado de la cooperación entre las firmas. Esta literatura se centra en las aglomeraciones geográficas como entornos de innovación y aprendizaje en donde puede emerger una "cultura de aprendizaje" (Cooke, 1998). Las actividades de coordinación de la producción en toda la cadena de valor resultan en procesos de aprendizaje que pueden dar lugar a innovaciones que aumentan la competitividad de la región. Patchell (1996) menciona el surgimiento de "habilidades relacionales" que se desarrollan a partir de las relaciones de cooperación entre las firmas nucleares y sus subcontratistas. Esta últimas van especializándose y ajustando su producción a las demandas de las firmas nucleares, mientras estas 
se adaptan a la demanda externa, y mediante un proceso sostenido de cooperación y coordinación, se logra construir habilidades que aumentan la competitividad del complejo, que son específicas a la organización industrial de la cadena y que mantienen un grado de flexibilidad. En otras palabras:

Las habilidades relacionales específicas contribuyen al upgrading de la región al abrir oportunidades para los subcontratistas de incrementar la demanda de sus bienes mediante transacciones con otras firmas nucleares, y la oportunidad a estas firmas nucleares de aprovechar el desarrollo de sus subcontratistas, con la posibilidad de expandir hacia nuevos sistemas productivos. (Patchell, 1996, p. 492)

Estos procesos permiten a la región pasar del learning by doing al learning by learning (Cooke, 1998, p. 17-18). El énfasis en la cualidad aprendiente de las redes de cooperación institucionalizadas que se tejen en los clústeres también se menciona Raco (1999). Este aspecto de aprendizaje colectivo caracteriza también a la escuela del milieux innovador (grupo GREMI), de acuerdo con la clasificación de Newlands (2002, p. 525).

Sin embargo, se encontraron autores que matizan esta relación establecida entre la innovación, las aglomeraciones geográficas, las actividades intensivas en tecnología y la cooperación, particularmente con respecto al sesgo hacia industrias de alta tecnología (Lundvall, 2007, p. 9). En su revisión del caso de desarrollo de industrias intensivas en tecnología en Finlandia, Ornston (2014) observa que, capitalizando sobre las redes de cooperación heredadas del periodo posguerra, el éxito de la estrategia tuvo como resultado un resquebrajamiento de la cooperación entre firmas. Para explicar este desenlace, Ornston (2014) apunta a dos factores. Primero, la naturaleza de estas industrias las vuelve vulnerables a innovaciones disruptivas que destruyen capacidades previamente construidas; y segundo, la naturaleza altamente competitiva de estas industrias exacerbó la competencia indeseada descrita anteriormente (Ornston, 2014, p. 460). Así, a pesar de que estas redes de cooperación (no solo entre firmas sino entre empleadores y trabajadores, y con actores estatales también) posibilitaron el desarrollo exitoso de industrias de alta tecnología en Finlandia, resultaron ser a su vez, el precio a pagar. Las temáticas abordadas en este trabajo matizan la relación cooperación-innovación-dinamismo tecnológico, a su vez que resaltan otros factores importantes a tomar en cuenta caso por caso, como la naturaleza de la industria específica en cuestión.

\subsection{Factores externos al distrito o clúster}

Una dimensión que se encuentra en casi todos los trabajos revisados es el efecto que pueden tener factores externos sobre las relaciones de cooperación entre las firmas. Rabellotti (1997) menciona la importancia de no considerar la cooperación o los acuerdos que la posibilitan como instituciones invariables en el tiempo. Por ejemplo, en su análisis del distrito industrial del valle de Sino en el sur del Brasil, Schmitz identifica cambios en las formas y niveles de cooperación entre firmas debido a factores externos (Schmitz, 1995, p. 547). Así mismo, Lorenz menciona el resquebrajamiento de la confianza y las redes de cooperación en los distritos industriales de Saint-Étienne (Francia), los distritos industriales alemanes, y en los distritos indus- 
triales de Birmingham y Sheffield (Gran Bretaña) (Sabel \& Zeitlin,1985; Herringel, 1990 en Lorenz, 1992, p. 179).

Al respecto, Raco (1999) menciona una crítica aguda a la literatura de los Nuevos Distritos Industriales. Esta literatura se centra en los aspectos endógenos de la región para explicar su éxito, ignorando aquellos aspectos externos de economía política nacional o incluso global que puedan jugar un papel en explicar el desarrollo desigual en un país o región; así, constituyen solo una explicación parcial del proceso (Raco, 1999, p. 963).

Enfocarse en la proactividad local ignora los contextos más amplios en los que las localidades operan, y así el desarrollo de capacidades institucionales locales meramente las encierran aún más en un espiral vicioso de competencia por inversiones globales móviles (Harvey, 1989; Leitner, 1991; Kantor, 1995 en Raco, 1999, p. 963).

Un ejemplo de competencia por inversiones nacionales móviles se observa en Gordon (2007).

\section{Conclusiones}

Las conceptualizaciones teóricas de la cooperación en la literatura de desarrollo regional pueden resumirse en cuatro dimensiones de acuerdo con las temáticas más relevantes identificadas en la discusión.

- La organización industrial compuesta de relaciones verticales y horizontales entre las firmas y cuya complejidad amerita la cooperación entre ellas.

- La necesidad de un equilibrio entre cooperación y competencia en las relaciones entre firmas para mantener la ventaja competitiva de la región. Dentro de esta dimensión se da un énfasis a los procesos de aprendizaje e innovación que emergen de la cooperación.

- La necesidad de un trasfondo cualitativo que permita la emergencia y existencia de redes de confianza y mecanismos de gobernanza que permitan el control social de los miembros de la región, propiciando la reciprocidad y adherencia a los códigos acordados.

- El carácter dinámico de las relaciones de cooperación y el efecto que pueden tener los factores externos sobre su mantenimiento.

\section{Referencias}

Acs, Z. J., \& Fitzroy, F. R. (1989). Inside the firm and organizational capital: A review article. International Journal of Industrial Organization, 7(2), 309-314. https://doi.org/https://doi. org/10.1016/0167-7187(89)90026-X

Acs, Z. J., \& Audretsch, D. B. (1990). Small Firms in the 1990s. En D. B. Acs, Zoltan J. y Audretsch (Ed.), The Economics of Small Firms: A European Challenge (pp. 1-24). Springer Science+ Business Media Dordrecht. https://doi.org/10.1007/978-94-015-7854-7

Amin, A. (1994). Post-Fordism: Models, Fantasies and Phantoms of Transition. En A. Amin (Ed.), Post-Fordism: A Reader (pp. 1-41). Oxford: Blackwell Publishers. https://doi.org/10.1002/ 9780470712726 
Arku, G. (2014). Competition and Cooperation in Economic Development: Examining the Perceptions of Practitioners in Ontario, Canada. Journal of Urban Affairs, 36(1), 99-118. https://doi.org/10.1111/j.1467-9906.2012.00647.x

Axelrod, R. (1984). The evolution of cooperation. Nueva York: Basic Books Inc.

Carlsson, B. (1989). The evolution of manufacturing technology and its impact on industrial structure: An international study. Small Business Economics, 1(1), 21-37. https://doi. org/10.1007/BF00389914

Cooke, P. (1998). Introduction: Origins of the Concept. En H.J. Braczyk, P. C. Cooke, y M. Heidenreich (Eds.), Regional Innovation Systems: the role of governances in a globalized world (pp. 2-26). UCL Press. https://doi.org/10.4324/9780203390702_chapter_1

Cox, K. R., \& Wood, A. (1997). Competition and Cooperation in Mediating the Global: The Case of Local Economic Development. Competition \& Change, 2(1), 65-94. https://doi. org/10.1177/102452949700200102

Felzensztein, C. (2008). Clusters, social networks and marketing collaboration in small firms: exploratory evidence from Chile and Scotland. International Journal of Entrepreneurship and Small Business, 6(2), 230-244. https://doi.org/10.1504/IJESB.2008.01863

Goetz, E. G., \& Kayser, T. (1993). Competition and Cooperation in Economic Development: A Study of the Twin Cities Metropolitan Area. Economic Development Quarterly, 7(1), 6378. https://doi.org/10.1177/089124249300700106

Gordon, V. (2007). Partners or Competitors? Perceptions of Regional Economic Development Cooperation in Illinois. Economic Development Quarterly, 21(1), 60-78. https://doi. org/10.1177/0891242406291573

Kamnungwut, W., \& Guy, F. (2012). Knowledge in the air and cooperation among firms: Traditions of secrecy and the reluctant emergence of specialization in the ceramic manufacturing district of Lampang, Thailand. Environment and Planning A, 44(7), 1679-1695. https://doi.org/10.1068/a44522

Lorenz, E. H. (1992). Trust, Community, and Cooperation: Toward a theory of industrial districts. En M. Storper y A. J. Scott (Eds.), Pathways to Industrialization and Regional Development (pp. 175-182). Nueva York: Routledge. https://doi.org/https://doi. org/10.4324/9780203995549

Loveman, G., \& Sengenberger, W. (1991). The Re-Emergence of Small-Scale Production: An International Comparison. Small Business Economics, 3(1), 1-37. https://doi.org/https:// doi.org/10.1007/BF00389842

Lundvall, B. (2007). National Innovation Systems. Analytical Concept and Development Tool. Industry and Innovation, 14(1), 95-119. https://doi.org/10.1080/13662710601130863

Neto, J. A. (2008). Productive cooperation network as a competitive advantage for small and medium firms in the Brazilian state of São Paulo. International Journal of Entrepreneurship and Small Business, 5(2), 201-211. https://doi.org/https://doi.org/10.1504/ IJESB.2008.016593

Newlands, D. (2003). Competition and Cooperation in Industrial Clusters: The Implications for Public Policy. European Planning Studies, 11(5), 521-532. https://doi.org/10.1080/096 54310303649

Nowak, M. A. (2006). Evolutionary dynamics: Exploring the Equations of Life. Belknap Press of Harvard Univ. Press. https://doi.org/10.2307/j.ctvjghw98

Ornston, D. (2014). When the High Road Becomes the Low Road: The Limits of High-Technology Competition in Finland. Review of Policy Research, 31(5), 454-477. https://doi. org/10.1111/ropr.12091

Patchell, J. (1996). Kaleidoscope Economies: The Processes of Cooperation, Competition, and Control in Regional Economic Development. Annals of the Association of American Geographers, 86(3), 481-506. https://doi.org/10.1111/j.1467-8306.1996.tb01763.x 
Piore, M., \& Sabel, C. F. (1984). The Second Industrial Divide: Possibilities for Prosperity. Nueva York: Basic Books Inc.

Porter, M. E. (1980). Competitive Strategy: Techniques for Analyzing Industries and Competitors (Republishe). Nueva York: The Free Press.

Porter, M. E. (1990). The Competitive Advantage of Nations. Harvard Business Review. Recuperado de: https://bit.ly/2HeRKIt

Rabellotti, R. (1997). External Economies and Cooperation in Industrial Districts: A Comparison of Italy and Mexico. Londres: Macmillan Press Ltd. https://doi.org/10.1007/978-1-349-25794-2

Raco, M. (1999). Competition, Collaboration and the New Industrial Districts: Examining the Institutional Turn in Local Economic Development. Urban Studies, 36(5-6), 951-968. https://doi.org/https://doi.org/10.1080/0042098993295

Sabel, C. F. (1994). Flexible Specialization and the Re-emergence of Regional Economies. En A. Amin (Ed.), Post-Fordism: A Reader (pp. 101-156). https://doi.org/https://doi. org/10.1002/9780470712726.ch4

Sayer, A. (1989). Postfordism in question. International Journal of Urban and Regional Development, 13, 666-695. https://doi.org/https://doi.org/10.1111/j.1468-2427.1989.tb00141.x

Schmitz, H. (1995). Collective efficiency: Growth path for small-scale industry. The Journal of Development Studies, 31(4), 529-566. https://doi.org/10.1080/00220389508422377

Thornberry, J. B. (2002). Competition and Cooperation: A Comparative Analysis of SEMATECH and the VLSI Research Project. Enterprise \& Society, 3(4), 657-686. https://doi. org/10.1017/S1467222700011976

Trapido, D. (2007). Competitive Embeddedness and the Emergence of Interfirm Cooperation. Social Forces, 86, 165-191. https://doi.org/10.1353/sof.2007.0110 